\title{
Investigating the response of Arabidopsis thaliana and Solanum lycopersicum to multiple simultaneous stresses
}

\author{
N. J. Atkinson ${ }^{1}$, C. Orfila ${ }^{2}$ and P. E. Urwin ${ }^{1}$ \\ ${ }^{1}$ Centre for Plant Sciences, Faculty of Biological Sciences, University of Leeds, Leeds LS2 9JT, UK and ${ }^{2}$ School of Food \\ Science and Nutrition, Faculty of Mathematics and Physical Sciences, University of Leeds, Leeds LS2 9JT, UK
}

Biotic and abiotic stresses are major factors adversely affecting yield and nutrient levels in crops worldwide. Although field conditions are likely to present more than one environmental stress at any time, traditionally each stress and its effect on a plant have been studied independently. To fully understand the nature of plant response to stress, it is therefore important to study stress factors in combination.

The molecular response of the model plant Arabidopsis thaliana to combined biotic and abiotic stress was investigated using wholegenome transcriptomics. Plants were subjected to dehydration stress, infection by the plant-parasitic nematode Heterodera schachtii, or both stresses simultaneously. Microarray analysis was carried out in triplicate using ATH1 Affymetrix chips, and the number of genes differentially regulated between treatments was determined. In roots, 427 genes were found to be significantly up-regulated by the two stresses in combination but not by either stress individually. In leaves, 472 such genes were identified. These data suggest that certain genes may specifically control the response of plants to multiple stresses, perhaps to allow conservation and prioritisation of resources. Further investigation of such genes may present opportunities for the engineering of crop plants with enhanced broad-spectrum stress tolerance.

The effects of combined stress on the physiology and nutritional qualities of the tomato Solanum lycopersicum are currently being investigated. Plants have been exposed to drought or infection by the nematode Meloidogyne incognita, as well as the stresses in combination. Sugar, carotenoid and flavonoid content is currently being analysed by HPLC, and ethylene content by gas chromatography. 